\title{
Projetos de Modulações sobre Superfícies via Sistema Integrado de Transmissão de Dados ${ }^{1}$
}

J.D. LIMA², Departamento de Matemática e Estatística, DME, UERN, Cx.P. 70, 59633-010, 15054-000 Mossoró, RN, Brasil.

R. PALAZZO Jr., Departamento de Telemática, DT, UNICAMP, Cx.P. 6101, 13081-970 Campinas, SP, Brasil.

Resumo. Em um sistema integrado de transmissão de sinais [3], os componentes canal-modulação-codificação são projetados de forma dependentes, isto é, o projeto de modulação é o modelo topológico do mergulho de 2-células, sobre uma superfície $\Omega$, do grafo associado ao canal e, o sistema de codificação é extraído de uma estrutura algébrica, o grupo de homologia de $\Omega$. Neste trabalho, identificaremos modelos de modulações com padrões do sistema integrado, destacando aqueles com características de espaços de sinais geometricamente uniformes [2].

Palavras-chave. Modulação, canal discreto sem memória, mergulho de grafo.

\section{Introdução}

Um sistema de transmissão de sinais se diz integrado [3], quando o projeto de modulação é sobre um mergulho de 2-células do grafo associado ao canal sobre uma superfície $\Omega$ e a codificação é extraída diretamente de uma estrutura algébrica associada ao modelo deste mergulho (o grupo de homologia de $\Omega$ [3]). O propósito é eliminar distorções causadas pela escolha inadequada desses componentes. Em uma escolha aleatória, a modulação pode apresentar ambigüidades nas transições correspondentes ao canal, problema transferido ao projeto de codificação, exigindo do sistema, dispositivos adicionais para as correções de tais distorções.

Dado um grafo $C$ associado a um canal DMC (Discrete Memoryless Channel), existe um conjunto de superfícies sobre as quais é possível mergulhar $C$ como um mergulho de 2-células [6]. Obtido um modelo de mergulho de um grafo, tem-se, necessariamente, um projeto topológico de modulação. Algumas famílias de grafos, como os completos, biparticionados e triparticionados, possuem mergulhos mínimo e máximo "conhecidos", ou seja, sabe-se quem são seus sistemas de rotações e gêneros da superfícies sobre as quais $C$ pode ser mergulhado, informações necessárias, mas não suficientes para se construir o mergulho de fato.

\footnotetext{
${ }^{1}$ Trabalho financiado pelo Programa Primeiros Projetos, MCT/CNPq/SINTEC 002/2003.

2jddeus@uol.com.br

${ }^{3}$ palazzo@dt.fee.unicamp.br
} 
Se o problema é construir o modelo planar ou espacial de um mergulho em que nada se sabe, em princípio, do gênero da superfície e do sistema de rotações, neste caso, o processo de construção é totalmente aleatório. Contudo, se é conhecido os tipos de modulações existentes sobre uma determinada superfície, podemos usar o método de construção em [5] para obter um modelo de modulação. Uma vez que os grafos completos biparticionados $K_{m, n}$ são os modelos de grafos mais freqüêntes associados a canais DMC, os nossos objetivos são: relacionar os projetos de modulações para espaços de $n$ sinais, destacando às regulares, ou seja, àquelas que possuem características de espaços de sinais geometricamente uniformes (e.s.g.u.) [2], e identificar o número de modulações nos casos em que $n=2$ e $n=4$.

\section{Mergulhos em Superfícies com Bordos}

Mergulhos de grafos sempre se referem aos mergulhos em superfícies sem bordos. No entanto, será introduzido aqui o conceito de mergulho em superfícies com bordos e, como veremos em seguida, este conjunto contêm a maioria das modulações e.s.g.u.

No ambiente das variedades riemannianas, uma superfície de gênero $m$ com $r$ componentes de bordo é qualquer superfície homeomorfa a uma superfície de gênero $m$ menos $r$ pontos, onde cada ponto "retirado" representa, na superfície, a formação de uma componente de bordo, isto é, o bordo é uma curva homeomorfa a um círculo.

Como toda região de um mergulho de 2-células é homotópica a um ponto, topologicamente, a retirada de um ponto do interior de uma região, corresponde a remoção do seu interior. Assim mergulho com bordo será defindo como segue.

Definição 2.1. Seja $F_{m n} \equiv \Omega(\alpha)=\cup_{i=1}^{\alpha} R^{i}$ o modelo formado por a regiões geradas pelo mergulho do grafo $K_{m, n}$ sobre uma superfície compacta $\Omega$. Chamaremos de mergulho com $r$ componentes de bordo do grafo $K_{m, n}, r \leq \alpha$, sobre $\Omega_{r}$, um mergulho obtido pela eliminação de $r$ regiões de $F_{m n}$, denotado por $F_{m n}^{r}=\Omega_{r}(\alpha-r)=\cup_{i=1}^{\alpha-r} R^{i}$. Denominaremos o modelo $F_{m n} \equiv \Omega(\alpha)$ de mergulho de origem e, o modelo $F_{m n} \equiv \Omega_{k}(\alpha-k)$, de mergulho derivado.

Como no mergulho em superfície sem bordo $F_{m n} \equiv \Omega(\alpha)$, podemos remover $k$ regiões, $k=1,2, \cdots, \alpha$, então o número de mergulhos com bordos derivados é

$$
\bar{F}_{m n} \mid=\sum_{k=1}^{\alpha-1}\left(\begin{array}{l}
\alpha \\
k
\end{array}\right)=\alpha !
$$

Em um mergulho onde $\Omega(\alpha)$ contém $k$-regiões do mesmo tipo, pode-se remover $\alpha-k$ regiões para obter uma modulação e.s.g.u. sobre uma superfície com $k$ regiões idênticas e $\alpha-k$ componentes de bordos, isto é, uma modulação da forma $\Omega_{\alpha-k}(k)$. Assim, o número de modulações e.s.g.u. é bem maior no universo das superfícies com bordos, o que as torna uma importante fonte geradora de modulações e.s.g.u.

Dado uma modulação (o modelo planar $^{1}$ do mergulho) sobre uma superfície

\footnotetext{
${ }^{1} \mathrm{O}$ esquema do mergulho $C \hookrightarrow \Omega$ de um grafo $C$ sobre uma superfície $\Omega$, tanto pode ser construído sobre o polígono $n$-gon $\equiv \Omega$, quanto sobre o modelo espacial de $\Omega$. O modelo sobre $n$-gon é geralmente denominado de modelo planar.
} 
sem bordo $F_{m n} \equiv \Omega(\alpha)$, o mergulho derivado com $r$ componentes de bordos $F_{m n}^{r}=$ $\Omega_{r}(\alpha-r)$ é obtido aplicando-se sucessivamente $r$-vezes os seguintes procedimentos.

Construção 1. Escolha uma região $R_{i}=\left(p, p_{1}, \cdots, p_{i-1}\right)$ de $\Omega(\alpha)$. Então,

1. se $R_{i}$ possui um lado adjacente ${ }^{2}$ a $R_{i}$ em $p$ que intercepta o bordo $\rho$ do $n$-gon $\equiv$ em $p_{\rho}$, então,

i) translade, no sentido horário, os vértices $p, p_{1}, \cdots, p_{i-1}, p$ de $R_{i}$ para o bordo $\rho$, fazendo coincidir o primeiro $p$ da seqüência com o ponto $p_{\rho}$, criando uma componente de bordo $\left(p, p_{1}, \cdots, p_{i-1}, p\right)$ no interior de $\rho$;

ii) reproduza as demais conexões de $F_{m n}$ para obter o mergulho derivado com uma componente de bordo $F_{m n}^{1} \equiv \Omega_{1}(\alpha-1) \equiv \Omega(\alpha-1)-R_{i}$.

2. se $R_{i}$ não possui um lado adjacente interceptando o bordo de n-gon, translade vértices de $C$ até que um lado adjacente de $R_{i}$ intercepte o bordo de $n$-gon e aplique os procedimentos i) e ii) acima.

O translado de vértices consiste em mover um vértice e seus lados adjacentes, conservando o sistema de rotações. A Figura 1, contêm mergulhos mínimos nãoorientados do grafo $K_{4,4}$ associado ao canal 4-ário, $C_{4}$, com sistema de rotações

$$
\text { Rot }=\{0(1357), 1(1357), 2(0462), 3(0246), 4(1357), 5(0642), 6(1753), 7(0462)\} .
$$

Entenda por aplicações sucessivas da Construção 1, o ato de usar o mergulho sem bordo sobre $\Omega$ para construir um mergulho com uma componente de bordo $\Omega_{1}$; em seguida, usar $\Omega_{1}$ para obter um mergulho com duas componentes de bordos $\Omega_{2}$, e assim por diante, até obter o mergulho com $k$ compoentes de bordos, $\Omega_{k}$, a partir de $\Omega_{k-1}$. Os modelos na Figura 1, correspondem a mergulhos do grafo completo biparticionado $K_{4,4}$ sobre a superfície de Klein $K$ (garrafa de Klein), uma superfície não-orientada. Contêm os modelos planos e espaciais dos mergulhos $K_{4,4} \hookrightarrow K(8), K_{4,4} \hookrightarrow K_{1}(7)$ e $K_{4,4} \hookrightarrow K_{2}(6)$, todos com sistemas de rotações iguais a (2.2), obtidos através dos procedimentos da Construção 1. São mergulhos mínimos compostos por 8, 7 e 6 regiões, todas quadrangulares, e portanto, projetos topológicos sobre superfícies não-orientadas de modulações e.s.g.u.

\section{Modulações vindas de Grafos Biparticionado}

No processo de identificação de modulações usaremos os seguintes resultados:

1. Seja $G$ um grafo com $v$ vértices, $e$ lados e $f$ faces e $G \hookrightarrow \Omega$, um mergulho de 2-células, então vale a fórmula da característica de Eüler de $\Omega$ é dada por

$$
\chi(\Omega)=v-e+f .
$$

Para as superfícies compactas com $r$ componentes de bordos, temos

$$
\chi(\Omega)=\left\{\begin{array}{l}
2-2 m-r, \text { se } \Omega \equiv m T_{r} \\
2-m-r, \text { se } \Omega \equiv m P_{r} .
\end{array}\right.
$$

\footnotetext{
${ }^{2}$ Um lado $a$ de $C$ é adjacente a uma região $R_{i}$ em $p$ de $F_{m n} \equiv \Omega(\alpha)$ se, e somente se, $a$ está conectado ao vértice $p$ e não é um lado de $R_{i}$
} 


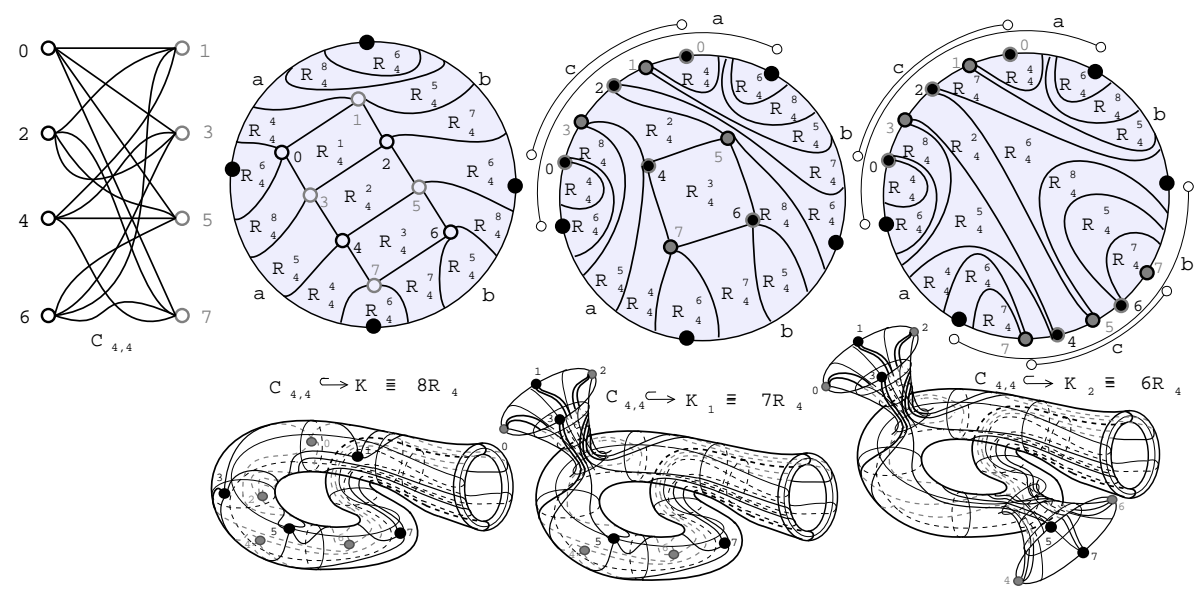

Figura 1: Mergulhos mínimos do canal 4-àrio: $C_{4,4} \hookrightarrow K, C_{4,4} \hookrightarrow K_{1}$ e $C_{4,4} \hookrightarrow K_{2}$

2. A característica ${ }^{1}$ do grafo completo biparticionado $K_{m, n}$ é dada por

$$
\gamma\left(K_{m, n}\right)=2[(m+n-m n / 2) / 2],
$$

onde $[\alpha]$ denota o maior inteiro menor ou igual ao número real $\alpha$.

3. O gênero mínimo [9] da superfície para o mergulho de $K_{4,4}$ é

$$
\gamma_{m}\left(K_{m, n}\right)=\{(m-2)(n-2) / 4\},
$$

onde $m, n \geq 2$ e $\{a\}$ denota o menor inteiro maior ou igual ao número real $a$;

4. O gênero máximo [7] da superfície para o mergulho de $K_{4,4}$ é

$$
\gamma_{M}\left(K_{m, n}\right)=[(m-1)(n-1) / 2],
$$

onde $m, n \geq 1$ e $[a]$ é o maior inteiro menor ou igual ao número real $a$;

5. Em toda superfície de gênero $\gamma$ tal que $\gamma_{m} \leq \gamma \leq \gamma_{M}$, existe um mergulho de 2-células de $K_{m, n}$ [7];

6. Existe uma correspondência biunívoca entre cada sistema de rotações de um grafo $G$ e um mergulho de 2-células de $G$ [6].

O conjunto das modulações sobre $\Omega$ vindas de mergulhos do grafo $K_{m, n}$ será denotado por $\mathbb{M}_{m, n}$, se $\Omega$ é orientada e por $\overline{\mathbb{M}}_{m, n}$, se $\Omega$ é não-orientada. Das igualdades (3.4), (3.5) e ítem 4. dos resultados acima, deduzimos imediatamente que

$$
\mathbb{M}_{m, n}=\left\{\gamma_{m} T,\left(\gamma_{m}+1\right) T, \cdots, \gamma_{M} T\right\} .
$$

\footnotetext{
${ }^{1}$ Chama-se característica do grafo $G$, o inteiro $\gamma(G)$ correspondente ao valor máximo de $\chi(\Omega)$ dentre todas as superfícies orientadas $\Omega$ nas quais $G$ tem um mergulho de 2-células
} 
Para o caso não-orientado $\overline{\mathbb{M}}_{m, n}$, basta trocar, na fórmula (3.6), $T$ por $P$.

Temos que $\left|\mathbb{M}_{m n}\right|=\gamma_{M}\left(K_{m, n}\right)-\gamma_{m}\left(K_{m, n}\right)+1$, onde $|A|$ é o número de elementos do conjunto $A$. Pondo $m=4 t+a, n=4 s+b, a, b \in\{0,1,2,3\}$, resulta que

$\left|\mathbb{M}_{m n}\right|=\left\{\begin{array}{l}m n / 4, \text { se }(m, n) \equiv(0,0), \cdots,(0,3),(1,0), \cdots,(3,0),(2,2) \bmod 4 \\ m n / 4+1 / 2, \text { se }(m, n) \equiv(1,2),(2,1),(2,3),(3,2) \bmod 4 \\ m n / 4+1 / 4, \text { se }(m, n) \equiv(1,3),(3,1) \bmod 4 \\ m n / 4-1 / 4, \text { se }(m, n) \equiv(1,1),(3,3) \bmod 4\end{array}\right.$

onde $m, n \equiv\left(a_{1}, b_{1}\right), \cdots,\left(a_{s}, b_{s}\right)(\bmod 4)$ indica que $m \equiv a_{1}(\bmod 4)$ e $n \equiv b_{1}(\bmod 4)$, $\cdots, m \equiv a_{s}(\bmod 4)$ e $n \equiv b_{s}(\bmod 4)$.

Em particular, para o grafo $K_{n, n}$, temos que

$$
\left|\mathbb{M}_{n, n}\right|=\left\{\begin{array}{l}
n^{2} / 4, \text { se }, n \equiv 0 \bmod 2 \\
n(n+2) / 4, \text { se } n \equiv 1 \bmod 2
\end{array}\right.
$$

\subsection{Tipos de modulações}

Os fatores que diferenciam uma modulação da outra são: a quantidade de sinais (igual ao número de regiões do mergulho), tipos de regiões de Voronoy, sistema de rotações de $K_{m, n}$ e o tipo de superfície $\Omega$ onde se encontra $K_{m, n}$ mergulhado.

Quanto às regiões de Voronoy, as modulações vindas de grafos $K_{m, n}$ apresentam sempre regiões com um número par de lados $\geq 4$, ou seja, para $t \in \mathbb{Z}$, têm-se

$$
R_{i} \in K_{m, n} \hookrightarrow \Omega \Rightarrow i=2 t+2, t \geq 2 .
$$

Por outro lado, da fórmula de Eüler (3.1) e da característica de $K_{m, n}$ em (3.3), segue que o número de regiões $\alpha$ de $\mathfrak{F}_{m n}$ é dado por

$$
\alpha=2[(m+n-m n / 2) / 2]+m n-m-n .
$$

Escrevendo $m=4 k+a$ e $n=4 t+b, a, b \in\{0,1,2,3\}$ e substituindo todos os casos possíveis para $m$ e $n$ na expressão de $\alpha$ em (3.10), obtemos

$\alpha=\left\{\begin{array}{l}\frac{m n}{2}, \text { se }(m, n) \equiv(0,0),(0,2),(1,2),(2,0) \cdots,(2,3),(3,2) \bmod 4 ; \\ m n / 2-1, \text { se }(m, n) \equiv(0,1),(0,3),(1,0),(3,0) \bmod 4 ; \\ m n / 2+1 / 2, \text { se }(m, n) \equiv(1,3),(3,1) \bmod 4 ; \\ m n / 2-3 / 2, \text { se }(m, n) \equiv(1,1),(3,3) \bmod 4,\end{array}\right.$

onde $m, n \equiv\left(a_{1}, b_{1}\right), \cdots,\left(a_{s}, b_{s}\right) \bmod 4$.

De uma análise em (3.11) deduzimos que:

i) Se $K_{n, n} \hookrightarrow \Omega(\alpha)$ é um mergulho mínimo, então,

$$
\alpha=\left\{\begin{array}{l}
n^{2} / 2, \text { se } n \equiv 0 \bmod 2 \\
n^{2} / 2-3 / 2, \text { se } n \equiv 1 \bmod 2 .
\end{array}\right.
$$

ii) O número de regiões de $K_{m, n} \hookrightarrow \Omega(\alpha)$ é par se, e somente se, $m$ e $n$ são pares.

iii) O número de regiões de $K_{n, n} \hookrightarrow \Omega(\alpha)$ é par se, e só se, $n$ é um inteiro par. 


\section{Modulações com $\alpha$ Sinais}

O mergulho $\mathfrak{F}_{m n} \equiv \Omega(\alpha)$ será denominado de modulação para um espaço de $\alpha$ sinais. O conjunto das modulações para espaço de $\alpha$ sinais será indicado por

$$
\left.\mathfrak{M}_{\alpha}=\left\{\mathfrak{F}_{m n}: \mathfrak{F}_{m n} \equiv \Omega(\alpha) \text { e } K_{m, n} \hookrightarrow \Omega\right\}\right\}
$$

onde $\mathfrak{F}_{m n}=\bigcup_{j=1}^{\alpha} R_{i_{j}}, i_{1} \leq \cdots \leq i_{\alpha}$ e $R_{i_{j}}$ é uma região com $i_{j}$ lados de $K_{m, n} \hookrightarrow \Omega_{\alpha}$.

Como $K_{m, n}$ tem $m n$ lados e $m n$ é par, então, a cardinalidade de $\mathfrak{M}_{\alpha}$ é igual ao número de soluções inteiras positivas da equações

$$
2 m n=4 F_{4}+6 F_{6}+8 F_{8}+\cdots e \sum_{i=0} F_{4+2 i}=\alpha .
$$

Sendo $\mathfrak{F}_{m n}=\cup_{j=1}^{\alpha} R_{i_{j}}$, segue imediatamente de (4.2) que $\sum_{j=1}^{\alpha} i_{j}=2 m n$, ou seja,

$$
\mathfrak{F}_{m n}=R_{i_{1}} \cup \cdots \cup R_{i_{2}} \Rightarrow i_{1}+\cdots+i_{\alpha}=2 m n .
$$

Se $\mathfrak{F}_{2}^{i}=R_{i 1} \cup R_{i 2}$ é uma modulação sobre $\Omega$, então, por (4.3), $i_{1}+i_{2}=2 m n$. Como $i_{1}, i_{2} \in\{4,6, \cdots, m n\}$, então existem $m n / 2-1$ modelos de modulações para $\mathfrak{F}_{2}$. Logo, por (4.3), as modulações com duas regiões são da forma seguinte.

Teorema 4.1. $\mathfrak{M}_{2}$ tem mn/2-1 modulações. Mais precisamente,

$$
\mathfrak{M}_{2}=\left\{\mathfrak{F}_{m n}^{1}, \mathfrak{F}_{m n}^{2}, \cdots, \mathfrak{F}_{m n}^{k}, \cdots, \mathfrak{F}_{m n}^{m n / 2-2}\right\}
$$

onde $\mathfrak{F}_{m n}^{1}=R_{4} \cup R_{2 m n-4}, \cdots, \mathfrak{F}_{m n}^{\frac{m n}{2}-2}=R_{m n-2} \cup R_{m n+2}, \mathfrak{F}_{m n}^{\frac{m n}{2}-1}=R_{m n} \cup R_{m n}$.

\section{Modulações com 4 Sinais}

Exibiremos somente a forma algébrica e o número de modulações existentes. Como forma algébrica de $\mathfrak{F}_{4}$ depende apenas do número de lados de $K_{m, n}$ e da fórmula de Eüler (3.1), não se sabe se o mergulho $K_{4,4} \hookrightarrow \Omega(4)$ é realizado em uma superfície orientada ou não-orientada. No modo algébrico, a modulação $\mathfrak{F}_{4}$ tem a forma

$$
\mathfrak{F}_{4}=R_{4+2 i_{1}} \cup R_{4+2 i_{2}} \cup R_{4+2 i_{3}} \cup R_{4+2 i_{4}}
$$

onde $i_{j} \in\{1,2,3, \cdots\}$, para todo $j=1,2,3,4$ e $i_{1} \leq i_{2} \leq i_{3} \leq i_{4}$.

Por (5.1), o máximo de $4+2 i_{j}$ é no caso de $j=4$ na quádrupla $(4,4,4,4+$ $\left.2 i_{4}\right)$. Logo, por $(4.2)$, devemos ter $4+2 i_{4}=m n / 2-6$. Assim, o problema consiste em determinar todas as combinações de quádruplas $\left(i_{1}, i_{2}, i_{3}, i_{4}\right)$, incluindo as com repetições, tais que, $i_{1} \leq i_{2} \leq i_{3} \leq i_{4}$ para todo $j \in\{1,2,3,4\}$ e $i_{j} \in$ $\{4,6,8, \cdots, m n / 2-6\}$. Para modulações com a primeira região fixa, vale o seguinte. 
Proposição 1. Seja $\mathfrak{M}_{4}(\alpha)$ o conjunto de modulações de $\mathfrak{M}_{4}$ que fixam a primeira região $i_{1}=\alpha$. Se $\alpha=4,6$, então,

$$
\begin{aligned}
\left|\mathfrak{M}_{4}(4)\right|= & \left\{\begin{array}{l}
\sum_{\substack{t=0 \\
m n / 6-8 / 3}}^{m / 6-2}(4+6 t), \text { se } m n \equiv 0(\bmod 3) \\
\sum_{t=0}^{m n / 6-7 / 3}(5+6 t)+m n / 2-3, \text { se } m n \equiv 1(\bmod 3) \\
\sum_{t=0}^{m}(3+6 t)+m n / 2-3, \text { se } m n \equiv 2(\bmod 3)
\end{array}\right. \\
\left|\mathfrak{M}_{4}(6)\right|= & \left\{\begin{array}{l}
\sum_{\substack{t=0 \\
m n / 6-8 / 3}}^{m / 6-3}(3+6 t)+m n / 2-5, \text { se } m n \equiv 0(\bmod 3) \\
\sum_{t=0}^{m n / 6-7 / 3}(4+6 t), \text { se } m n \equiv 1(\bmod 3) \\
\sum_{t=0}^{m}(5+6 t)+m n / 2-5, \text { se } \operatorname{mn} \equiv 2(\bmod 3)
\end{array}\right.
\end{aligned}
$$

Os procedimetos usados na obtenção das fórmulas (5.2) e (5.3) também aplicamse aos demais casos. A variação do índice $i_{3}$ controla a quantidade de subconjuntos distintos de $\mathfrak{M}_{4}$ cujos índices $i_{1}$ e $i_{2}$ são fixos.

\subsection{Seqüência dos tipos de modulações com 4 sinais}

No cálculo do número de modulações, as seqüências dos números de modulações que fixam a primeira região, representa um fator decisivo na questão da contagem.

Definição 5.1. Seja $\mathfrak{I}_{1}=\{4,6, \cdots, m n / 2\}$ o conjunto da variação do índice $i_{1}$. Seja $\mathfrak{M}_{4}(\mu)=\left\{\mathfrak{F}_{m n} \in \mathfrak{M}_{4}: \mathfrak{F}_{m n}=R_{\mu} \cup R_{i_{2}} \cup R_{i_{3}} \cup R_{i_{4}}, \mu \in \mathfrak{I}_{1}\right\}$ o conjunto de todos os modelos de $\mathfrak{M}_{4}$ que fixam $i_{1}=\mu$. Chamaremos de seqüência dos números de modelos que fixam $i_{1}$ a seqüência $S_{t}(\mu)=\left(a_{0}, a_{1}, \cdots, a_{t}\right), a_{k}=\left|\mathfrak{M}_{4}(4+2 k)\right|$.

A ordem nos índices estabelecida em (5.1), induz necessariamente uma interdependência entre os mesmos a qual será estabelecida a seguir.

Definição 5.2. Se $K_{m, n} \hookrightarrow \Omega$ e $\mathfrak{F}_{4} \equiv \Omega(\alpha)$, indicaremos por $\mathfrak{I}_{s}, s \in\{1,2, \cdots, \alpha\}$, o conjunto dos índices da região $R_{s}$ de um modelo $\mathfrak{F}_{4} \in \mathfrak{M}_{\alpha}$. Se $s>1$, chamaremos de índice mínimo relativo $i_{s \min }^{\text {rel }}$ o menor índice do conjunto $\mathfrak{I}_{s}$ relativo aos indices anteriores. Chamaremos de indice máximo relativo $i_{s \max }^{\text {rel }}$ o maior índice de $\mathfrak{I}_{s}$ relativo aos demais indices.

Denotamos por $\mathfrak{I}_{2}^{\mu}$ o conjunto dos índices da segunda região de um modelo relativo à primeira região $i_{1}=\mu$. Neste caso, $i_{2 \min }^{\mu}$ é o índice mínimo para a segunda região relativa à primeira. Chamaremos de $i_{3}^{\mu, \eta}$ o índice da terceira região relativa às duas primeiras. Observe que o conjunto dos índices da $s$-ésima região é $\mathfrak{I}_{s}=\left\{4,6,8, \cdots, i_{s \max }\right\}$. 
Das definições e resultados anteriores, deduz-se facilmente as propriedades enunciadas da seguinte forma:

Proposição 2. Se $\mathfrak{F}_{4}^{i}=R_{i_{1}} \cup R_{i_{2}} \cup R_{i_{3}} \cup R_{i_{4}} \in \mathfrak{M}_{4}$, então,

i) $i_{1 \max }=m n / 2, i_{2 \max }=[(2 m n-4) / 3], i_{3 \max }=m n-4, i_{4 \max }=m n-6$;

ii) $i_{s \min }^{r e l}=i_{s-1} e i_{s \max }^{r e l}=\left(2 m n-\sum_{j=1}^{s-1} i_{j}\right) /(\alpha-s+1)$;

iii) O número de modulações com 4 regiões do conjunto $\mathfrak{M}_{4}$ é $\left|\mathfrak{M}_{4}\right|=\sum_{k=0}^{m n / 4-1} a_{k}$;

iv) O número de termos da seqüência $S_{t}(\mu)$ é $\mathrm{mn} / 4$.

v) $a_{k}=\sum_{t=0}^{[(m n-4 k-8) / 3]}([(m n-3 t) / 2]-3-2 k)$.

As formas dos modelos de modulações que fixam as duas primeiras regiões podem ser deduzidas de (5.1), (5.2) e Proposição 2(ii), conforme o enunciado da seguinte:

Proposição 3. As modulações de $\mathfrak{M}_{4}(\mu, \eta)$ assumem uma das duas formas:

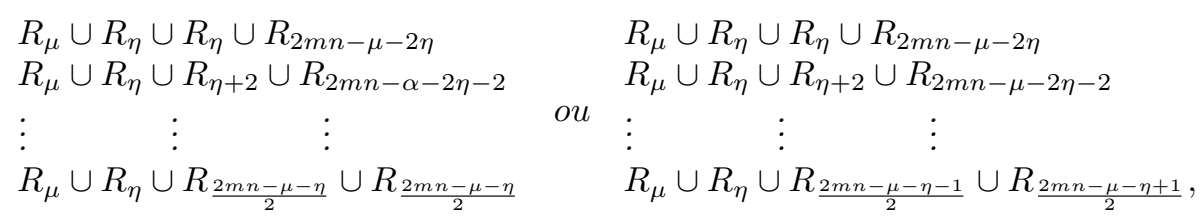

caso $k$ seja par ou ímpar, respectivamente.

Proposição 4. Seja $S_{h}(\mu, \eta)=\left(b_{0}, b_{1}, \cdots, b_{h}\right), b_{j}=\left|\mathfrak{M}_{4}(\mu, \mu+4 j)\right|$, a seqüência de números de elementos do conjunto $\mathfrak{M}_{4}(\mu, \eta)$ que fixam $i_{1}=\mu$ e $i_{2}=\eta$, então

$$
h=\left\{\begin{array}{l}
\sum_{\mu \in \mathfrak{I}_{1}}(m n-2 \mu) / 3, \text { se } m n \equiv p(\bmod 3), \mu \equiv q(\bmod 3) \text { e } p+q \equiv 0(\bmod 3), \\
\sum_{\mu \in \mathfrak{I}_{1}}(m n-2 \mu-1) / 3, \text { semn } \equiv p(\bmod 3), \mu \equiv q(\bmod 3) \text { e } p+q \equiv 1(\bmod 3), \\
\sum_{\mu \in \mathfrak{I}_{1}}(\operatorname{mn}-2 \mu-2) / 3, \text { se } m n \equiv p(\bmod 3), \mu \equiv q(\bmod 3) e p+q \equiv 2(\bmod 3) .
\end{array}\right.
$$

Substituindo $\mu$ por $4-2 j$ nas expressões de $h$ da Proposição 4 , e analisando os termos da seqüência $S_{h}(\mu, \eta)$, deduzimos os seguintes resultados.

Corolário 5.0.1. Se $S_{h}(\mu, \eta)=\left(b_{0}, b_{1}, \cdots, b_{h}\right)$ é a seqüência de números de elementos do conjunto de modelos $\mathfrak{M}_{4}(\mu, \eta)$ que fixam $i_{1}=\mu$ e $i_{2}=\eta$, então,

i) $O$ índice de variação $h$ e o k-ésimo termo de $S_{h}(\mu, \eta)$, são:

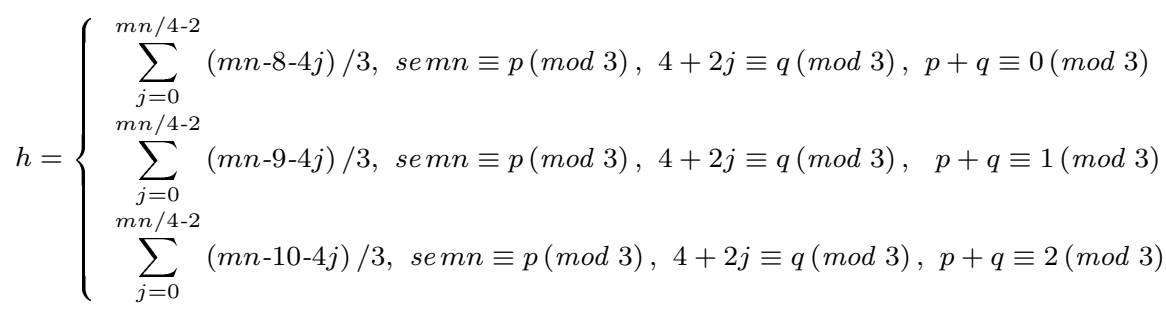




$$
b_{k}=\left\{\begin{array}{l}
(m n-2 \mu-3 k) / 2+1, \text { se } k \equiv 0(\bmod 2) \\
(m n-2 \mu-3 k-1) / 2+1, \text { se } k \equiv 1(\bmod 2) .
\end{array}\right.
$$

ii) As seqüêcias $S_{h}^{0}(\mu, \eta)=\left(b_{0}, b_{2}, \cdots, b_{2 p}\right)$ e $S_{h}^{1}(\mu, \eta)=\left(b_{1}, b_{3}, \cdots, b_{2 p+1}\right)$, dos termos pares e ímpares de $S_{h}(\mu, \eta)$, são progressões aritméticas decrescentes de razão 3.

Escrevemos $(m, n, p) \equiv(r, s, t)(\bmod 3)$ para significar que $m \equiv r(\bmod 3), n \equiv$ $s(\bmod 3)$ e $p \equiv t(\bmod 3)$.

Assim, a cardinalidade de $\mathfrak{M}_{4}(\mu, \eta)$ é a soma dos elementos da seqüência $S_{h}(\mu, \eta)$.

Teorema 5.1. Se $m n \equiv p(\bmod 3)$ e $\mu \equiv q(\bmod 3)$, então as seqüências de números de modulações do conjunto $\mathfrak{M}_{4}(\mu, \eta)$ que fixam $\mu \in \mathfrak{I}_{1}, \eta \in \mathfrak{I}_{2}$ é

$S_{h}(\mu, \eta)=\left\{\begin{array}{l}(m n / 2-\mu+1, m n / 2-\mu-1, m n / 2-\mu-2, \cdots, 8,7,5,4,2,1), \text { se } p+q \equiv 0(\bmod 3) \\ (m n / 2-\mu+1, m n / 2-\mu-1, m n / 2-\mu-2, \cdots, 9,7,6,4,3,1), \text { se } p+q \equiv 1(\bmod 3) \\ (m n / 2-\mu+1, m n / 2-\mu-1, m n / 2-\mu-2, \cdots, 9,8,6,5,3,2), \text { se } p+q \equiv 2(\bmod 3) .\end{array}\right.$

Demonstração. Pondo $m n \equiv p(\bmod 3)$ e $\mu \equiv q(\bmod 3)$, analisando os 9 casos possíveis e aplicando Corolário 5.0.1(v).

Aplicando, ao Teorema 5.1, a Proposição 2(v) e (5.4), resulta o seguinte

Corolário 5.1.2. Se $a_{k} \in S_{t}(\mu)$, então $a_{k}=\sum_{\eta \in \mathfrak{I}_{2}^{4+2 k}} b_{h}, b_{h} \in S_{h}(4+2 k, \eta)$.

Os termos de $S_{t}(\mu)$ são obtidos de $S_{h}(\mu, \eta)$ segundo a relação $a_{k}=\sum_{\eta \in \mathfrak{I}_{2}^{4+2 k}} a_{h}$, $a_{h} \in S_{h}(4+2 k, \eta)$. Pelo Corolário 5.1.2, $a_{k}$ é a soma dos termos $b_{k}$ 's da seqüência $S_{h}(\mu, \eta)$, cuja soma será obtida do arranjo triangular da Tabela 1.

Tabela 1: Triângulo $\nabla$

$\begin{array}{cccccccccc}a_{h_{4}}^{4} & \cdots & a_{7}^{4} & a_{6}^{4} & a_{5}^{4} & a_{4}^{4} & a_{3}^{4} & a_{2}^{4} & a_{1}^{4} & a_{0}^{4} \\ \vdots & \vdots & \vdots & \vdots & \vdots & \vdots & \vdots & \vdots & \vdots & \\ 11 & 9 & 8 & 6 & 5 & 3 & 2 & & & \\ 9 & 7 & 6 & 4 & 3 & 1 & & & & \\ 7 & 5 & 4 & 2 & 1 & & & & & \\ 5 & 3 & 2 & & & & & & & \\ 3 & 1 & & & & & & & & \\ 1 & & & & & & & & & \end{array}$




\subsection{Número das modulações para 4 sinais oriundas de $K_{m, n}$}

A cardinalidade de $\mathfrak{M}_{4}(\mu)$ é igual ao somatório dos elementos de $\nabla$.

Teorema 5.2. Se $m n \equiv p(\bmod 3)$ e $\mu \equiv q(\bmod 3)$, então o número de elementos de $\mathfrak{M}_{4}(\mu)$ é igual a

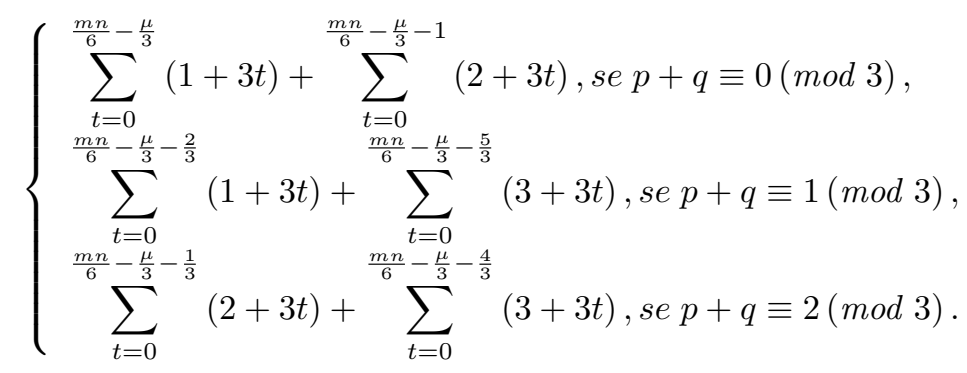

Demonstração. Conseqüências do Corolário 5.0.1 e Teorema 5.1.

Do Teorema 5.2, segue a forma simplificada do próximo corolário.

Corolário 5.2.3. Se $m n \equiv p(\bmod 3)$ e $\mu \equiv q(\bmod 3)$, a classe de congruência módulo 3, então o número de elementos do conjunto $\mathfrak{M}_{4}(\mu)$, de modelos com 4 regiões que fixam a primeira região $\mu$, é igual a

$$
\begin{aligned}
& \sum_{t=0}^{\frac{m n}{6}-\frac{\mu}{3}-1}(3+6 t)+m n / 2-\mu+1, \text { se } p+q \equiv 0(\bmod 3) \\
& \frac{m n}{6}-\frac{\mu}{3}-\frac{2}{3} \\
& \sum_{t=0}^{6}(4+6 t), \text { se } p+q \equiv 1(\bmod 3) \\
& \sum_{t=0}^{\frac{m n}{6}-\frac{\mu}{3}-\frac{4}{3}}(5+6 t)+m n / 2-\mu+1, \text { se } p+q \equiv 2(\bmod 3) \text {. }
\end{aligned}
$$

Teorema 5.3. O número de elementos de $\mathfrak{M}_{4}$ é

$$
\left|\mathfrak{M}_{4}\right|=\left\{\begin{array}{l}
\sum_{\substack{i=1 \\
m n / 4-1}}^{m} i^{2}+\sum_{\substack{i=1 \\
m n / 12-4 / 3}}^{m n / 12-1}(3 i)^{2}, \text { se } m n \equiv 0(\bmod 3) \\
\sum_{\substack{i=1 \\
m n / 4-1}}^{m} i^{2}+\sum_{\substack{i=0 \\
m n / 12-5 / 3}}^{m}(1+3 i)^{2}, \text { se } m n \equiv 1(\bmod 3) \\
\sum_{i=1}^{m} i^{2}+\sum_{i=0}^{2}(2+3 i)^{2} \text { se } m n \equiv 2(\bmod 3) .
\end{array}\right.
$$

Demonstração. Segue da soma dos elementos da Tabela 1.

A Tabela 2 contém o número de modulações de $\mathfrak{M}_{4}, m \in\{2,4, \cdots, 12\}$ e $n \in$ $\{4,6, \cdots, 10\}$. E usando as fórmulas (3.1) e (3.2), identificamos, na Tabela 3, as modulações regulares com quatro sinais, ou seja, do tipo e.s.g.u. 
Tabela 2: Cardinalidade de $\mathfrak{M}_{4}$

\begin{tabular}{|c|cccc|}
\hline & $\mathbf{4}$ & $\mathbf{6}$ & $\mathbf{8}$ & $\mathbf{1 0}$ \\
\hline $\mathbf{2}$ & 1 & 5 & 15 & 34 \\
$\mathbf{4}$ & 15 & 64 & 169 & 351 \\
$\mathbf{6}$ & 64 & 249 & 632 & 1285 \\
$\mathbf{8}$ & 169 & 632 & 1575 & 3169 \\
$\mathbf{1 0}$ & 351 & 1285 & 3169 & 6336 \\
\hline
\end{tabular}

Tabela 3: Modulações e.s.g.u. de $\mathfrak{M}_{4}$

\begin{tabular}{|c|cccccccc|}
\hline$m$ & $n$ & $v$ & $e$ & $l$ & $K_{m, n}$ & $\chi(\Omega)$ & $\alpha T$ & $\beta P$ \\
\hline 2 & 3 & 5 & 6 & 4 & $K_{2,3}$ & -3 & $\varnothing$ & $\varnothing$ \\
2 & 4 & 6 & 8 & 4 & $K_{2,4}$ & 2 & $S$ & $\varnothing$ \\
2 & 5 & 7 & 10 & 4 & $K_{2,5}$ & 1 & $T$ & $P$ \\
2 & 6 & 8 & 12 & 4 & $K_{2,6}$ & 0 & $T$ & $2 P$ \\
2 & 7 & 9 & 14 & 4 & $K_{2,7}$ & -1 & $2 T$ & $3 P$ \\
2 & 8 & 10 & 16 & 4 & $K_{2,8}$ & -2 & 27 & $4 P$ \\
2 & 9 & 11 & 18 & 4 & $K_{2,9}$ & -3 & $\varnothing$ & $5 P$ \\
\hline
\end{tabular}

\section{Conclusão}

As modulações visadas atendem aos requisitos de um sistema integrado de transmissão de dados [3], os quais são planejados a partir do modelo de grafo associado ao canal DMC. Desse modo, o processo mais natural de identificação parte do canal e, por isso, o nosso objetivo foi relacionar os projetos de modulações para espaços de $\alpha$ sinais destacando às regulares, ou seja, àquelas com características e.s.g.u.

Se o objetivo é optar por uma particular modulação, a Proposição 3 irá dizer se ela existe e, a Tabela 3, identifica a superfície sobre a qual o projeto é realizado. Quanto a construção, o processo em [5] encarrega-se do mergulho em superfície sem bordos e a Construção 5.0.1, do mergulho em superfície com bordos.

O número de elementos de $\mathfrak{M}_{4}$ na Tabela 2 , já é expressivo, mas não é só isto, da condição (2.1), segue que o número de modulações em superfícies com bordos é 24 vezes maior do que o apresentado na Tabela 1.

\section{Agradecimentos}

À Maria Zuleica Oriá Lima (esposa) e ao Prof. Elder Lacerda Queiroz, Faculdade de Filosofia - UERN, pelas assessorias prestadas no texto e na tradução.

\footnotetext{
Abstract. In a transmission system of digital signals [3], the components channelmodulation-codification are projected by the following way: the modulation is the topological model of 2-cells embedding on surface $\Omega$ of complete bipartite graphs
} 
$K_{m, n}$, associated to the discrete memoryless channels. The main focus is to identify the modulation models with integrated systems patterns, emphasizing the geometrically uniform signal spaces [2].

Keywords. Modulations, discrete memoryless channel, surface, graph embedding.

\section{Referências}

[1] P.A. Firby, C.F. Gardiner, "Surface Topology", Ellis Horwood Limited, England, 1991.

[2] G.D. Forney Jr., Geometrically Uniform Codes, IEEE Trans. Inform. Theory, 37 (1991) 1241-1260.

[3] J.D. Lima, "Identificação e Estrutura Algébrica das Superfícies Compactas com e sem Bordos, Provenientes de Mergulhos de Canais Discretos sem Memória", Tese de doutorado, FEEC, UNICAMP, 2002.

[4] J.D. Lima, R. Palazzo Jr., Grupo de homologia, uma fonte natural de códigos corretores de erros, em submissão, Mossoró, 2007.

[5] J.D. Lima, R. Palazzo Jr., Projetos topológicos de modulações sobre superfícies, em submissão, Mossoró, 2007.

[6] R.D. Ringeisen, Determining all compact orientable 2-manifolds upon which $K_{m, n}$ has 2-cell embeddings. Journal Combinatorial Theory, 12 (1972) 101-104.

[7] R.D. Ringeisen, Survey of results on the maximum genus of a graph, Journal of Graph Theory, 31979 1-13.

[8] H. Seifert, W. Threlfall, "Leciones de Topologia", Madrid, 1951.

[9] S. Stahl, A survey of the embeddings of a graph, Journal of Graph Theory, 2 (1978) 275-298.

[10] J.M. Wozencraft, I.M. Jacobs, "Principles of Comunication Engineering", New York, 1965. 